\title{
In-Situ Fabrication of Bi/BiMn-BiMn-Mn Graded Materials by High Magnetic Field Gradients
}

\author{
Tie LIU, ${ }^{1)}$ Qiang WANG, ${ }^{1)}$ Chunjiang WANG, ${ }^{1)}$ Zhigang LIU, ${ }^{1,2)}$ Yi YUAN ${ }^{11}$ and Jicheng HE ${ }^{1)}$ \\ 1) Key Laboratory of Electromagnetic Processing of Materials (Ministry of Education), Northeastern University, Shenyang \\ 110004 China. E-mail: wangq@epm.neu.edu.cn $\quad$ 2) Shenyang Junding Construction \& Decoration Engineering Co. Ltd., \\ Shenyang 110031 China.
}

(Received on May 24, 2010; accepted on September 30, 2010)

\begin{abstract}
In order to develop a new method for in situ fabricating multilayer functionally graded materials (FGMs), high magnetic field gradients are proposed to control the solidification behaviors of peritectic alloys. Solidification experiments of $\mathrm{Bi}-11.8 \mathrm{wt} \% \mathrm{Mn}$ alloys under various magnetic field gradient conditions have been conducted and the macro- and microstructures of the magnetic field-treated specimens were examined. The primary $\mathrm{Mn}$ and $\beta$ BiMn crystals can successively precipitate during the solidification process. Bi/BiMn-BiMn-Mn FGMs with various gradients have been successfully fabricated during solidification process. The results indicate that the gradient distribution of precipitates could be adjusted by controlling the solidification strategy and the magnetic parameters such as the product of the magnetic flux density and its gradient $B d B / d z$.
\end{abstract}

KEY WORDS: solidification; composite materials; high magnetic fields; functionally graded materials; intermetallic compounds; microstructure.

\section{Introduction}

Functionally graded materials (FGMs) are characterized by their spatially inhomogeneous composition and properties. To fabricate FGMs, a variety of methods have been developed. These methods include powder metallurgy, centrifugal casting, laser cladding, chemical deposition, and others. ${ }^{1-4)}$ However, all these traditional techniques are relatively expensive or too complicated to be used in industrial production. Therefore, it is necessary to explore a novel method for the fabrication of FGMs. For this purpose, Wang et al. ${ }^{5,6)}$ have successfully fabricated two kinds of FGMs using high magnetic gradients during the solidification and semi-solid processes based on the magnetoArchimedes levitation. ${ }^{7)}$ The present research was undertaken to further develop the technique of fabricating FGMs by high magnetic fields and to draw general conclusions for in situ fabrication of multilayer FGMs. A Bi-11.8wt\%Mn alloy was employed because it sequentially undergoes peritectic and eutectic reactions during the solidification process and two precipitations can be in situ produced from the melt. For brevity, the effects of uniform magnetic fields on the phase transformation, the magnetic property and the crystal orientation of $\mathrm{Bi}-\mathrm{Mn}$ alloys, which have been discussed by Yasuda, ${ }^{8)} \mathrm{Li}^{9)}$ and Wang, ${ }^{10)}$ are not involved in this study.

\section{Experimental}

A master alloy of $\mathrm{Bi}-11.8 \mathrm{wt} \% \mathrm{Mn}$ was prepared in a graphite crucible by melting bismuth and manganese with a purity of $99.5 \mathrm{wt} \%$ under a vacuum environment in an induction furnace. The quenched ingot was then cut into cylindrical specimens ( $9 \mathrm{~mm}$ in diameter, $15 \mathrm{~mm}$ in height) for the solidification experiments under high magnetic field gradient conditions. The experimental apparatus and the distribution of the magnetic field are both described in detail in a previous work. ${ }^{11)}$ The specimens were put into corundum crucibles of an inner diameter of $10 \mathrm{~mm}$ and the length of $50 \mathrm{~mm}$ and then set inside the bore of a superconducting magnet. Each specimen was heated in an Ar atmosphere to $1023 \mathrm{~K}$ at a heating rate of $5 \mathrm{~K} \cdot \mathrm{min}^{-1}$, and held at $1023 \mathrm{~K}$ for $20 \mathrm{~min}$ until it was completely melted. Then it was cooled down to $723 \mathrm{~K}$, which is slightly above the $\mathrm{L}+\mathrm{Mn} \rightarrow \beta \mathrm{BiMn}$ peritectic temperature of $719 \mathrm{~K}$, at a cooling rate of $10 \mathrm{~K} \cdot \mathrm{min}^{-1}$ and held for $20 \mathrm{~min}$ to make the primary $\mathrm{Mn}$ phases precipitate as much as possible. Then the specimen was cooled down to $623 \mathrm{~K}$ at a relatively low cooling rate of $5 \mathrm{~K} \cdot \mathrm{min}^{-1}$ and held for $30 \mathrm{~min}$ to ensure the complete migration of the BiMn phases. A holding time at $623 \mathrm{~K}$ is also designed for the removal of magnetic field to avoid the potential magnetic transformation from taking place at $613 \mathrm{~K}$, the Curie temperature of the BiMn phase. Finally, the specimen was cooled in a furnace to room temperature by turning off the power source of the furnace. The solidification experiment was repeated in the presence of an 8.6 T uniform magnetic field and three magnetic field gradients of $B \mathrm{~d} B / \mathrm{d} z=-50,-100$, and $-300 \mathrm{~T}^{2} / \mathrm{m}(B \mathrm{~d} B / \mathrm{d} z$ is the product of the magnetic flux density and its gradient and 8.6 T indicates the magnetic flux density at the specimen position). To obtain the above magnetic field conditions, the specimens were placed at the center and various 
positions above the center of the magnet bore, respectively. The magnetic field-treated specimens were cut longitudinally (parallel to the direction of the magnetic fields). The macro- and microstructures of the as-cast specimens were observed by a Leica-500 optical microscope.

\section{Results and Discussion}

Figure 1 shows some different kinds of solidified structures in $\mathrm{Bi}-11.8 \mathrm{wt} \% \mathrm{Mn}$ alloys. Figure 1(a) is the macrostructure of the specimen solidified without magnetic field. Three layers can be clearly distinguished throughout the whole section. In the upper layer, there are precipitated primary Mn crystals which are dark in the macrostructure. Another BiMn precipitated phase (gray) is in the middle layer. The lower layer is the metallic matrix of the $\mathrm{BiMn} / \mathrm{Bi}$ eutectic without any precipitates. The typical microstructures corresponding to the marked regions of each layer are shown in Fig. 2(a). Figure 1(b) shows the macrostructures
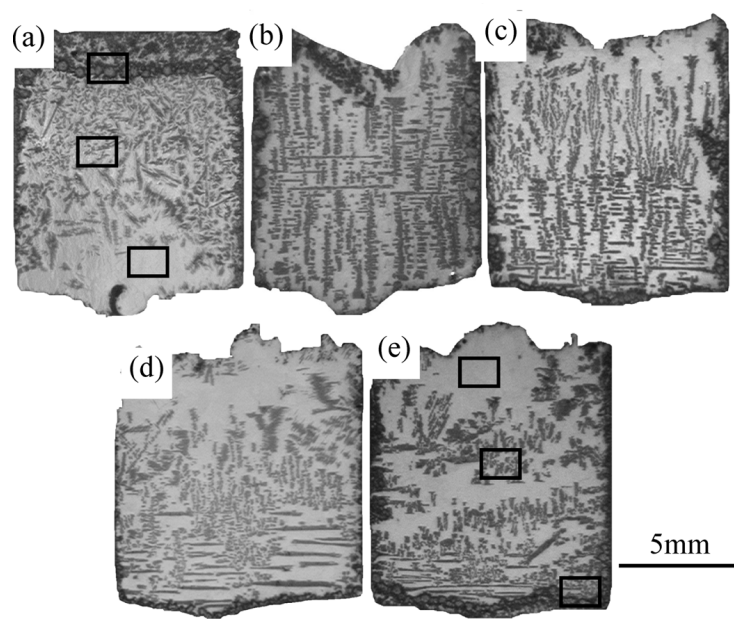

Fig. 1. Optical macrographs of $\mathrm{Bi}-11.8 \mathrm{wt} \% \mathrm{Mn}$ alloys solidified with and without an $8.6 \mathrm{~T}$ magnetic field with various magnetic field gradients (the magnetic field direction is vertical). (a) $B=0 \mathrm{~T}$; (b) $B=8.6 \mathrm{~T}$; (c) $B=8.6 \mathrm{~T}, B \mathrm{~d} B / \mathrm{d} z=$ $-50 \mathrm{~T}^{2} \cdot \mathrm{m}^{-1}$; (d) $B=8.6 \mathrm{~T}, B \mathrm{~d} B / \mathrm{d} z=-100 \mathrm{~T}^{2} \cdot \mathrm{m}^{-1}$; (e) $B=8.6 \mathrm{~T}, B \mathrm{~d} B / \mathrm{d} z=-300 \mathrm{~T}^{2} \cdot \mathrm{m}^{-1}$.

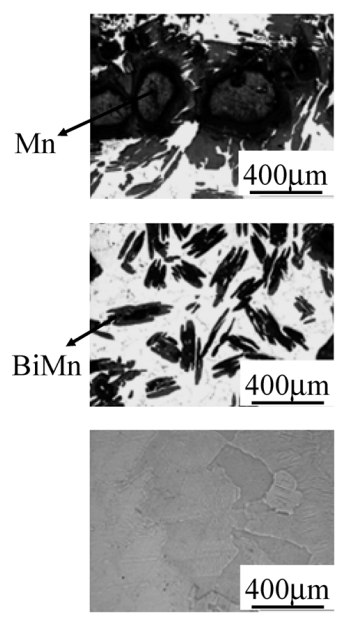

(a)
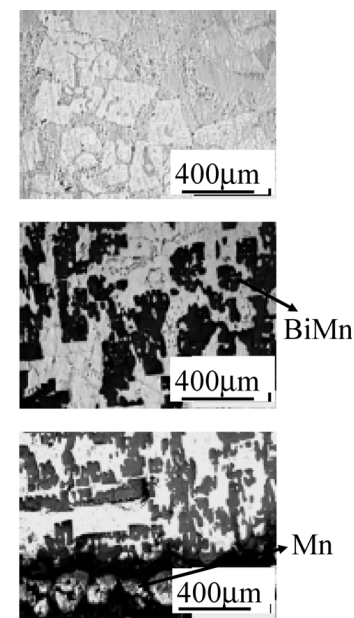

(b)

Fig. 2. Typical microstructures corresponding to the marked regions of each layer in Figs. 1(a) and 1(e), respectively. (a) $B=0 \mathrm{~T}$, (b) $B=8.6 \mathrm{~T}, B \mathrm{~d} B / \mathrm{d} z=-300 \mathrm{~T}^{2} \cdot \mathrm{m}^{-1}$. of the sample solidified under an 8.6 T uniform magnetic field. The primary BiMn phases are nearly homogeneously dispersed, although some primary Mn grains distribute mainly at the top and edge of the specimen just as the case of $B=0 \mathrm{~T}$. By increasing slightly the magnetic gradient $B \mathrm{~d} B / \mathrm{d} z$ to $-50 \mathrm{~T}^{2} \cdot \mathrm{m}^{-1}$ (the minus sign indicates the opposite direction to the magnetic field), an observable change will be detected on the macrostructure as shown in Fig. 1(c). The Mn grains mainly locate at the bottom and edge of the sample and BiMn grains remain homogeneously. When the magnetic gradient $B \mathrm{~d} B / \mathrm{d} z$ further increases to $-100 \mathrm{~T}^{2} \cdot \mathrm{m}^{-1}$, a graded structure with three layers is obtained, as shown in Fig. 1(d). It should be noted that the Mn grains disappear from the top of the specimen and gather mainly at the bottom of the specimen. The BiMn phases obviously tend to gather downward to the bottom of the sample. However, when a gradient magnetic field of $B=8.6 \mathrm{~T}$ and $B \mathrm{~d} B / \mathrm{d} z=-300 \mathrm{~T}^{2} \cdot \mathrm{m}^{-1}$ is imposed, the distribution of the three layer was completely reversed with that without magnetic field, as shown in Fig. 1(e). The typical microstructures corresponding to the marked regions of each layer in Fig. 1(e) are shown in Fig. 2(b). Therefore, it is expected that any desired distribution of the multiple phases could be achieved by adjusting the magnetic field conditions.

The distribution of the phases in solidified structures is determined by their migration behaviors. The mechanism of particles migrating in a melt under high magnetic fields has been described in detail in a previous work, ${ }^{11)}$ the main result of which is given as follows.

$$
v_{\mathrm{t}} \approx \frac{1}{3} \frac{\left(1 / \mu_{0}\right)\left(\chi_{\mathrm{s}}-\chi_{\mathrm{m}}\right) B(\mathrm{~d} B / \mathrm{d} z)-\left(\rho_{\mathrm{s}}-\rho_{\mathrm{m}}\right) g}{B \sqrt{\sigma_{\mathrm{m}} \eta_{0}}} r .
$$

where, $v_{\mathrm{t}}$ is the migration velocity, $\mu_{0}, \chi, \rho, \sigma, \eta_{0}$ are the vacuum permeability, volumetric magnetic susceptibility, density, electrical conductivity and viscosity, respectively. The subscripts $\mathrm{s}$ and $\mathrm{m}$ designate the solid particle and the melt. Equation (1) indicates that density, susceptibility, magnetic intensity and gradient are the dominant parameters for the migration behaviors.

According to the equilibrium phase diagram of the binary $\mathrm{Bi}-\mathrm{Mn}$ alloy, the melting point of a $\mathrm{Bi}-11.8 \mathrm{wt} \% \mathrm{Mn}$ alloy is about $915 \mathrm{~K}$ and the primary crystals $\mathrm{Mn}$ and $\beta$ BiMn can successively precipitate during the solidification process under the cooling conditions here adopted. When a magnetic field is absent, the precipitated Mn and BiMn, whose densities are both less than that of the melt, will float up and finally evolve into the graded structure of $\mathrm{Mn}-\mathrm{BiMn}-\mathrm{Bi} / \mathrm{BiMn}$. The migration velocities evidently decrease due to the suppression effect of the Lorentz force on a conducting fluid when a uniform magnetic field is imposed. Consequently, the distribution of both the primary $\mathrm{Mn}$ and $\beta \mathrm{BiMn}$ grains will be distributed relatively homogeneously throughout the specimen on the longitudinal section. In the case of a gradient magnetic field, the gravitational force, the magnetic force and the Lorentz force all contributed to the migration behavior. According to Eq. (1), the critical values of $-B \mathrm{~d} B / \mathrm{d} z$ for the levitation of $\mathrm{Mn}$ and BiMn phases are approximately calculated to be about $6 \mathrm{~T}^{2} \cdot \mathrm{m}^{-1}$ and $14 \mathrm{~T}^{2} \cdot \mathrm{m}^{-1}$, respectively. Here, the densities 


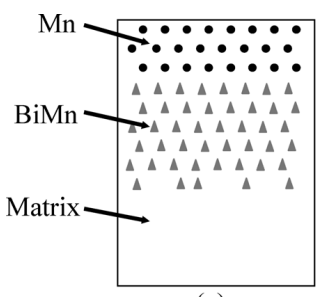

(a)

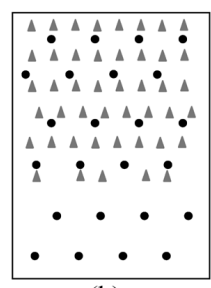

(b)

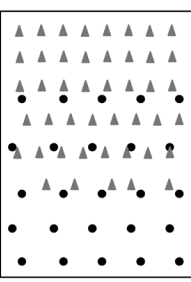

(c)

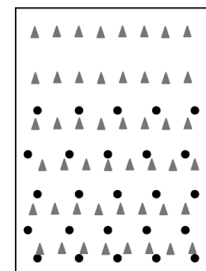

(d)

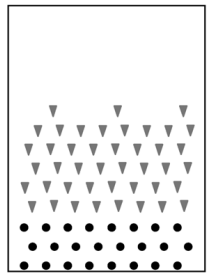

(e)
Fig. 3. Schematic illustration of the distributions of the primary $\mathrm{Mn}$ and BiMn under various magnetic field conditions. (a) Without magnetic field, both the primary $\mathrm{Mn}$ and BiMn are segregated in the upper part; (b) in the case of $-B \mathrm{~d} B / \mathrm{d} z \approx 6 \mathrm{~T}^{2} \cdot \mathrm{m}^{-1}$, the primary $\mathrm{Mn}$ is homogeneously distributed; (c) in the case of $6<-B \mathrm{~d} B / \mathrm{d} z<14 \mathrm{~T}^{2} \cdot \mathrm{m}^{-1}$, the primary $\mathrm{Mn}$ is segregated in the lower part; (d) in the case of $-B(\mathrm{~d} B / \mathrm{d} z) \approx 14 \mathrm{~T}^{2} \cdot \mathrm{m}^{-1}$, the primary BiMn is homogeneously distributed; (e) In the case of $-B \mathrm{~d} B / \mathrm{d} z \approx$ $14 \mathrm{~T}^{2} \cdot \mathrm{m}^{-1}$, both the primary $\mathrm{Mn}$ and BiMn are strongly segregated in the lower part.

and the magnetic susceptibilities of the $\mathrm{Mn}$, and $\mathrm{Bi}$ near their melting temperatures are 5.8 and $10.1 \times 10^{3} \mathrm{~kg} \cdot \mathrm{m}^{-312)}$ and 883 and $-6 \times 10^{-6},{ }^{13)}$ respectively. For $\beta \mathrm{BiMn}$, its density is $7.8 \times 10^{3} \mathrm{~kg} \cdot \mathrm{m}^{-3} \cdot{ }^{14)}$ Considering that it is paramagnetic, its magnetic susceptibilities is estimated to be $195 \times 10^{-6} .^{15)}$ So any graded distribution, as illustrated in Fig. 3, is theoretically realizable by adjusting properly the value of magnetic gradients. Unfortunately, all the values of $-B \mathrm{~d} B / \mathrm{d} z$ employed in this experiment are beyond the range of $1-38 \mathrm{~T}^{2} \cdot \mathrm{m}^{-1}$ due to the limitations of the homogeneity of the magnetic field, the size of the bore and other experimental conditions. Because of that, only two graded structures were fabricated.

It should be noted that the distribution of primary phases in Fig. 1 is somewhat different from that in the schematic illustrations in Fig. 3. First, some Mn grains appeared on the edge of specimens because of the combined action of the heat flux from melt to surroundings during the solidification process and the gradient of the magnetic field in the radial direction (for the magnetic field of $8.6 \mathrm{~T}$, the maximum value of $|B \mathrm{~d} B / \mathrm{d} r|$ in the radial direction covering a specimen with a diameter of $10 \mathrm{~mm}$ is about $\left.10 \mathrm{~T}^{2} \cdot \mathrm{m}^{-1}\right) .{ }^{11}$ ) Second, a small quantity of BiMn phases are also found in the Mn layers, which is the result of the fact that the BiMn phase can be produced by a peritectic reaction or precipitated directly from the melt. So the solidified structures and the graded distribution were also influenced by the characters of the system, as well as the solidification strategy.

\section{Conclusions}

$\mathrm{Bi} / \mathrm{BiMn}-\mathrm{BiMn}-\mathrm{Mn} \mathrm{FGMs}$ with various gradients have been successfully fabricated under magnetic field gradients. It indicates that the distribution of reinforcements in a matrix can be adjusted by controlling the magnetic field conditions. The proposed strategy is feasible to distribute multiple phases by systematically considering the characteristics of the system. Therefore, it presents a simple, but efficient, technique for the fabrication of multilayer FGMs by imposing a high magnetic field gradient during the solidification process.

\section{Acknowledgements}

This work was supported by the National Natural Science Foundation of China (Grant No. 51006020), the Fundamental Research Funds for the Central Universities (Grant Nos. N090209001, N090109001), the Program for New Century Excellent Talents in University (Grant No. NCET-06-0289) and the 111 Project (Grant No. B07015).

\section{REFERENCES}

1) G. Jin, M. Takeuchi, S. Honda, T. Nishikawa and H. Awaji: Mater. Chem. Phys., 89 (2005), 238.

2) Y. Watanabe and T. Nakamura: Intermetallics, 9 (2001), 33.

3) K. B. Panda and K. S. R. Chandran: Metall. Mater. Trans. A, 34 (2003), 1993.

4) R. Jedamzik, A. Neubrand and J. Roedel: J. Mater. Sci., 35 (2000), 477.

5) Q. Wang, T. Liu, A. Gao, C. Zhang, C. J. Wang and J. C. He: Scr. Mater, 56 (2007), 1087.

6) T. Liu, Q. Wang, A. Gao, C. Zhang, C. J. Wang and J. C. He: Scr. Mater., 57 (2007), 992.

7) Y. Ikezoe, N. Hirota, J. Nakagawa and K. Kitazawa: Nature, 393 (1998), 749.

8) H. Yasuda, I. Ohnaka, Y. Yamamoto, K. Tokieda and K. Kishio: Mater. Trans., 44 (2003), 2207.

9) X. Li, Z. M. Ren and Y. Fautrelle: Intermetallics, 15 (2007), 845.

10) Q. Wang, C. S. Lou, T. Liu, N. Wei, C. J. Wang and J. C. He: J. Phys. D: Appl. Phys., 42 (2009), 025001.

11) Q. Wang, C. J. Wang, T. Liu, K. Wang and J. C. He: J. Mater. Sci., 42 (2007), 10000.

12) D. J. Steinberg: Metall. Trans. A, 5 (1974), 1341.

13) R. Dupree and E. F. W. Seymour: Liquid Metals, Marcel Dekker, New York, (1972), 461.

14) J. B. Yang, W. B. Yelon, W. J. James, Q. Cai, S. Roy and N. Ali: J. Appl. Phys., 91 (2002), 7866.

15) R. R. Heikes: Phys. Rev., 99 (1955), 446. 